\title{
Executive function and extremely preterm children
}

\author{
Paul J. Chung ${ }^{1}$, Valerie P. Opipari ${ }^{2}$, Irene Koolwijk ${ }^{1}$ and the Pediatric Policy Council
}

$E^{*}$ xecutive function is a set of abilities that allow one to control one's behavior to achieve goals. These abilities include attention, impulse inhibition, working memory, and cognitive flexibility, and combine to allow for both planning and problem solving. Distinct from traditional conceptions of intelligence, executive function has been associated with longterm educational and health outcomes, and is likely an essential component of academic and social development.

In this issue of Pediatric Research, Heeren et al. present "Cognitive functioning at age 10 years among children born extremely preterm: a latent profile approach," which examines intelligence, executive function, and academic achievement in 87310 -year-old children born before 28 weeks' gestation in 11 cities in 5 states (1). Intelligence was measured using verbal and nonverbal reasoning scales; executive function was measured using working memory, attention, inhibition, and mental flexibility scales; and academic achievement was measured using both reading and math skill tests and parent-reported school problems (i.e., individualized education program, repeating a grade, and special education). In addition, the authors used latent profile analysis (LPA) to categorize children into empirical groups based on intelligence and executive function, comparing this categorization against traditional intelligence-only categories.

The results are interesting and worthy of attention. In this sample of extremely preterm (EP) children, LPA identified four groups. Overall, $34 \%$ fell into a category with the mean scores at about the norm, $41 \%$ fell into a category about $1 \mathrm{SD}$ below the norm (low-normal), 17\% fell into a category about 2 SDs below the norm (moderately impaired), and $8 \%$ fell into a category about 3-4 SDs below the norm (severely impaired). Traditional intelligence-only categories, however, identified $66 \%$ of children as unimpaired $\left(Z_{\mathrm{IQ}}\right.$ above -1$)$.

These differences suggest that incorporating executive function into assessments may identify children whose intelligence is normal but whose executive function deficits place them at risk. Moreover, that risk is substantial.

In the "low-normal" group (the large majority of whom would traditionally be considered "unimpaired"), $52 \%$ required an individualized education program, 21\% had repeated a grade, and $10 \%$ were in special education (vs. $28 \%$,
$7 \%$, and $3 \%$ of "normal" children, respectively). In other words, LPA identified a group of children who qualified as cognitively normal under traditional standards but who were, in fact, at high risk of poor academic outcomes. Without accounting for executive function, many children would only be identified once school failure was already occurring.

Interestingly, a separate study by a different group examining moderately preterm (32-36 weeks' gestation) children also found four categories using LPA, providing some evidence that these empirical groupings may, in fact, capture underlying constructs (2). Heeren et al. confirmed these constructs in EP children and reported a much higher prevalence of deficits and risk for school failure, as might be expected, given the difference in gestational age.

When executive function deficits and intellectual deficits are considered together, as they are in the LPA groupings, the life-long individual and societal burden of extreme prematurity becomes clear. Outside of complex chronic disease, the single most individually and societally costly childhood condition might be school failure. School failure is a threshold event, creating sudden and marked discontinuities in longterm economic and civic potential and productivity, thus predisposing individuals, and even subsequent generations, to early morbidity and mortality.

These results speak to an urgent need for large increases in both funding and mandated requirements for comprehensive and longitudinal developmental assessments, follow-up, and early intervention services for EP children. In the general population, need for early intervention services likely approaches 15\%, which makes universal screening with validated primary-care tools an appropriate strategy (3). In the EP population, however, Heeren et al. raise the possibility that up to two-thirds of children require some level of developmental services, which ironically makes universal screening less important and instead suggests the need for universal formal assessments by developmental specialists with longitudinal monitoring and fast-track access to services.

What is the current federal policy landscape regarding access to such care for EP children? Although multidisciplinary neonatal intensive care unit (NICU) follow-up clinics, which could be an ideal launching pad for intensive

\footnotetext{
${ }^{1}$ Department of Pediatrics, UCLA David Geffen School of Medicine and Mattel Children's Hospital, Los Angeles, California; ${ }^{2}$ Department of Pediatrics, University of Michigan Medical School, Mott Children's Hospital, Ann Arbor, Michigan.

Correspondence: Paul J. Chung (paulchung@mednet.ucla.edu)

Received 28 June 2017; accepted 27 July 2017; advance online publication 30 August 2017. doi:10.1038/pr.2017.184
} 


\section{Commentary $\mid$ Chung et al.}

developmental services, are recommended by the American Academy of Pediatrics and have proliferated in recent years, there is currently no mandate that all EP children be enrolled. Moreover, funding for NICU follow-up clinics typically ends at the age of 3 , which is just at the edge of viability for existing executive function-assessment tools. Likewise, executive function interventions are typically preschool/school-based, occurring after the age of 3 .

The magnitude of developmental concerns in EP children, the prevalence of executive function deficits specifically, and the primacy of academic achievement in shaping long-term health trajectories suggest the need for a new model of care. One option might be a new type of NICU follow-up clinic, one that expands to reach and accommodate all EP children, that extends all the way to kindergarten entry for children who demonstrate need, continues longitudinal ageappropriate developmental assessments that include executive functioning from the age of 3 onward, and is intimately connected with community and district preschools.

Grants provided to states under Part C of the Individuals with Disabilities Education Act (IDEA) may provide one pathway for identifying EP children in need of intervention. Part $\mathrm{C}$ of the IDEA provides money to states to maintain statewide systems to provide for early intervention services for infants and toddlers with disabilities, developmental delays, or those who are at risk for developmental delays. Provisions in Part $\mathrm{C}$ require that services focus on children from birth through age 2 , although children with disabilities at the age of
3 and over may continue to receive Part C early intervention services until they are eligible to enter kindergarten. With enhanced Part C funding, states could prioritize the identification of EP children as those in need of continued monitoring and services, creating a universal system of longitudinal monitoring to identify executive functioning delays up to the point when the child enters school. The Title V Maternal and Child Health Services Block Grant Program is a critical partner in this effort through its work on outreach, identification, service coordination, and parental involvement. With enhanced Title V funding, linkages between Part C and Title V for EP children could be strengthened. Such a model would need new federal requirements and funding to support it, but there is ample reason to believe that this investment might be worth it.

Disclosure: The authors declare no conflict of interest.

\section{REFERENCES}

1. Heeren T, Joseph RM, Allred EN, O'Shea TM, Leviton A, Kuban KCK. Cognitive functioning at age 10 years among children born extremely preterm: a latent profile approach. Pediatr Res 2017 (doi:10.1038/pr.2017.82; e-pub ahead of print).

2. Cserjesi R, Van Braeckel KN, Timmerman M, et al. Patterns of functioning and predictive factors in children born moderately preterm or at term. Dev Med Child Neurol 2012;54:710-5.

3. Rosenberg SA, Zhang D, Robinson CC. Prevalence of developmental delays and participation in early intervention services for young children. Pediatrics 2008;121:e1503-9. 